\title{
Inclusión digital y educación inclusiva. Aportes para el diseño de proyectos pedagógicos con el uso de tecnologías de la comunicación'
}

\author{
Sandra Acevedo Zapata ${ }^{2}$
}

\begin{abstract}
Resumen
Este documento presenta algunas reflexiones sobre la inclusión digital como estrategia para conseguir la inclusión social acompañada de la educación inclusiva, estrategias necesarias en la formación de maestros. Estas reflexiones son el producto de un proceso investigativo sobre el estado del arte de lo que se ha trabajado frente a la inclusión digital y la educación inclusiva, en la educación superior en el periodo comprendido entre el año 2000 hasta el año 2014, el cual se realizó como investigación documental, identificando los avances en este campo y los aportes que se suscitan para la formación de maestros, y brinda elementos concretos sobre el diseño de proyectos pedagógicos con inclusión digital.
\end{abstract}

Palabras clave: inclusión social, tecnologías de la comunicación, educación inclusiva, aprendizaje, inclusión digital.

\footnotetext{
1. Agradecimiento: Especial reconocimiento a Gina Torres Amaya, por el trabajo documental realizado, soporte de este artículo.

2. Docente de planta - UNAD. Licenciada en Psicología y Pedagogía con títulos de postgrado en Dirección Estratégica de Organizaciones Universitarias, Gestión Social de la Educación y Maestría en Educación de la Universidad Pedagógica Nacional. Doctorando en Ciencias Gerenciales. Con experiencia como investigadora en el campo de la pedagogía y las tecnologías de la comunicación para la educación inclusiva. Investigación en procesos cognitivos para la construcción de pensamiento prospectivo.

Correo: sandra.acevedo@unad.edu.co,sacevedoz@yahoo.es

ORCID: http://orcid.org/0000-0003-0518-0234
} 


\title{
Digital inclusion and inclusive education. Contributions to design educational projects with the use of communication technologies
}

\begin{abstract}
This paper presents some thoughts on digital inclusion as a strategy to achieve social inclusion accompanied by inclusive education, which are strategies that need to be integrated in the training of teachers. These thoughts are the product of a research process on the state of the art of what has worked against digital inclusion and inclusive education in higher education in the period from 2000 to 2014 , which is he performed as documentary research, identifying progress in this field and the contributions that arise for teacher training, and provides concrete elements for the design of educational projects with digital inclusion.
\end{abstract}

Keywords: Social inclusion, communication technologies, inclusive education, learning, digital inclusion.

Recibido: 13-03-2014

Aceptado: 06-05-2014

\section{Introducción}

Teniendo en cuenta que la inclusión social es el propósito de la sociedad y objetivo del milenio, se han desarrollado diversas estrategias, entre ellas la educación inclusiva y la inclusión digital, en esta última se encuentra implícito el uso y apropiación de las tecnologías de la comunicación en pos de generar mejores condiciones de acceso y equidad en la calidad de vida de todos los sujetos de las diversas poblaciones que integran la sociedad.

Por tanto para comprender cómo se implementan estas estrategias, se desarrolló un proceso investigativo entre el año 2013 y 2014, el cual fue financiado por la Subdirección de apoyo a las Instituciones de Educación Superior del Ministerio de Educación Nacional. Fruto de este trabajo, surgen algunas reflexiones, que pueden ser tenidas en cuenta en el diseño de proyectos pedagógicos, desde la perspectiva de la educación inclusiva con el uso de las tecnologías de la comunicación, lo cual puede permitir la configuración de proyectos de inclusión digital que puedan aportar a la inclusión social. 


\section{Marco referencial: la educación inclusiva nociones fundamentales}

\section{Evolución de la noción inclusión}

Es importante comprender que la inclusión digital y la educación inclusiva son estrategias centrales para conseguir construir la inclusión social, la cual requiere ser comprendida superando la dicotomía tradicional asociada al concepto de exclusión, lo que permite pensar en un modelo educativo abierto y generoso, que entiende la diversidad como una característica inherente de la vida y que trabaja en todos sus sistemas para conseguir atenderla de manera adecuada.

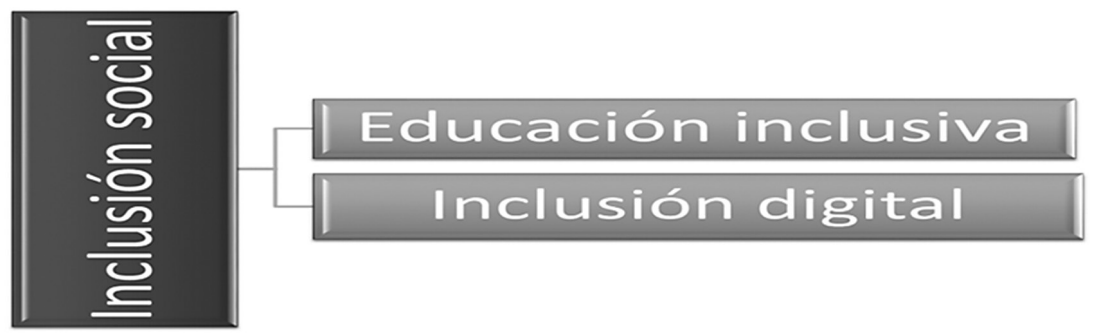

Gráfico 1. Categorías fundamentales de inclusión social (Acevedo, 2014).

Se ha dado un cambio de paradigma de la educación, la mirada convencional, que la redujo a grupos de población y personas categorizadas en función de las denominadas necesidades especiales, y de situaciones de marginalidad y de pobreza, debe ampliarse y transformarse en un principio/eje transversal de organización y de funcionamiento de los sistemas educativos.

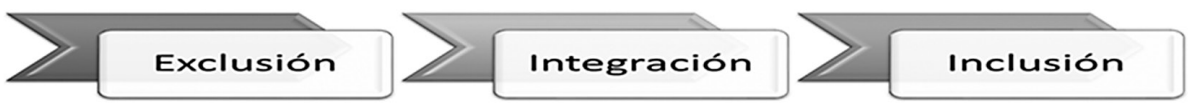

Gráfico 2. Evolución de categorías sobre inclusión (Acevedo, 2014).

La exclusión es una primera etapa educativa en la cual la escuela solo se encargaba de preparar a la población urbana y burguesa, donde las mujeres, los campesinos, las personas pertenecientes a la clase trabajadora y todos aquellos grupos pertenecientes a las culturas marginales, eran excluidas de todo proceso educativo.

Después se da el cambio a las reformas integradoras, las cuales generan un cambio en el reconocimiento del derecho a la educación. «En esta fase de reformas integradoras, las políticas educativas que se mantienen sectorizadas por grupos de población comparten el reconocimiento de la igualdad de oportunidades ante 


\section{Sandra Acevedo Zapata}

Inclusión digital y educación inclusiva. Aportes para el diseño de proyectos pedagógicos con el uso de tecnologías de la comunicación. Artículo de reflexión.

la educación, pero limitando esa igualdad únicamente al acceso a la educación. En ningún modo se garantiza el derecho a recibir respuestas a las propias necesidades desde la igualdad y mucho menos la igualdad de metas» (Parrilla, 2002: 17).

Los procesos de integración de personas con necesidades educativas especiales (NEE) inician el reconocimiento del derecho a la educación para dicha población, luego se da un proceso de integración centrada en la normalización y las instituciones educativas intentaron impartir sus valores desde la perspectiva de la cultura dominante.

En la búsqueda de un reconocimiento de derechos, en todo su esplendor para todas las personas, nace la inclusión con dos grandes características: por un lado dichos procesos inclusivos no son únicamente para personas con NEE, sino para «todas las personas» y por otra parte la inclusión «significa participar en la comunidad de todos en términos que garanticen y respeten el derecho, no sólo a estar o pertenecer, sino a participar de forma activa política y civilmente en la sociedad, en el aprendizaje en la escuela» (Parrilla, $2002: 8$ ).

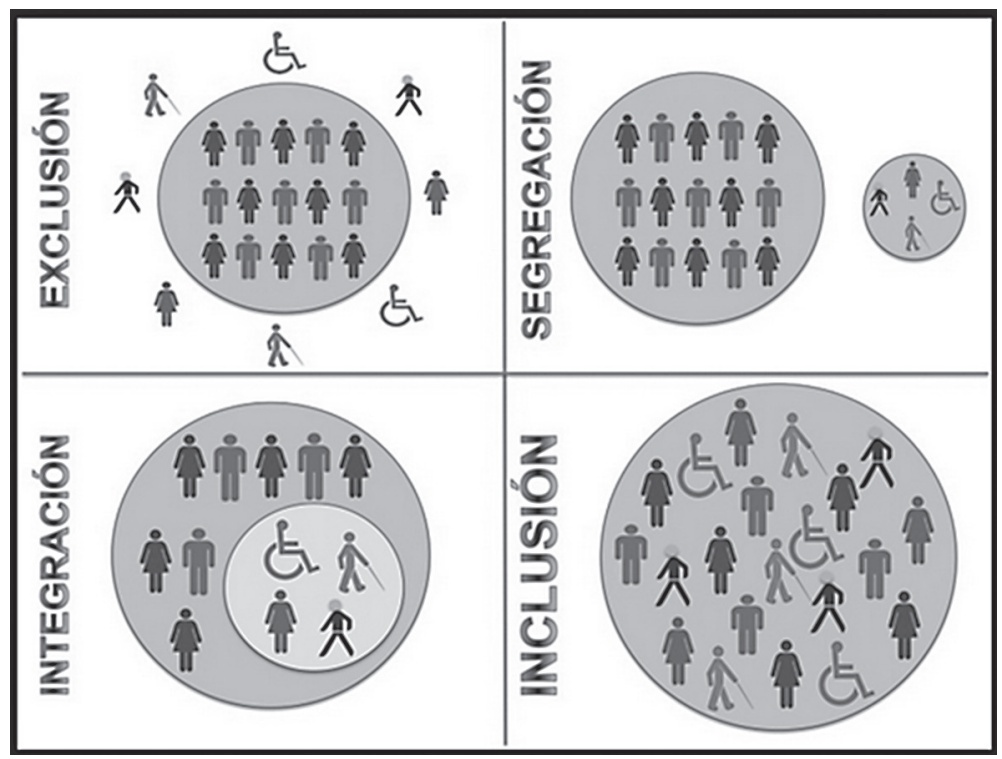

Fuente: Fernández, C y otros. En IANA 2012. 
La más reciente respuesta ofrecida por las instituciones educativas ante la diversidad, es la inclusión, la cual consiste en esa garantía para todas las personas de no solo pertenecer y estar en un sitio sino participar de forma activa. La inclusión se plantea en consecuencia como un derecho humano garantizando así una equidad social.

En la última etapa se consolida la educación inclusiva como categoría académica, ésta ha evolucionado hacia una perspectiva que da cuenta de los procesos de formación personalizada, que se brinda a los sujetos en su diversidad de expectativas y necesidades.

Para la educación inclusiva se toma como referencia fundamental que: «La inclusión parte del supuesto que todo ser humano es especial a su manera, y que requiere una respuesta educativa singular que transforme el potencial de aprendizaje que todas y todos abrigamos, en una realidad gratificante y sustentable» ( MEN, 2013).

Desde esta perspectiva de inclusión, se hace necesario repensar las propuestas pedagógicas de las instituciones y de los maestros, centrando su accionar a partir de las necesidades de los sujetos y sus procesos de aprendizaje en la diversidad de posibilidades.

\section{Fundamentos epistemológicos en la educación inclusiva}

El compromiso con la formación de sujetos, desde la perspectiva de la estrategia de educación inclusiva, implica preguntarse y cuestionar los esquemas de organización del currículo, que principalmente se basan en una sumatoria de asignaturas cargadas de contenidos, en los cuales se encuentra una desconexión con los contextos y problemáticas que han creado las necesidades y son significativos para una formación relevante y pertinente de los sujetos.

Requiere también cuestionar e investigar sobre la práctica docente, lo que implica diseñar proyectos pedagógicos con el propósito de trasformar las metodologías tradicionales de enseñanza, centradas en transmitir conocimiento y que en muchos casos se aleja de las expectativas y necesidades de los sujetos, de la sociedad y de las culturas.

Para conseguir la inclusión educativa que es el propósito esencial de las sociedades democráticas, con la regla de defender y proteger los Derechos Humanos, la educación inclusiva ha ido adquiriendo un lugar relevante en el mundo desde su primera aparición durante la Conferencia Mundial sobre necesidades educativas especiales en Salamanca en 1994, allí se dio el cambio del paradigma anterior, 


\section{Sandra Acevedo Zapata}

Inclusión digital y educación inclusiva. Aportes para el diseño de proyectos pedagógicos con el uso de tecnologías de la comunicación. Artículo de reflexión.

centrado en la integración de las personas con discapacidad, hacia el nuevo, orientado a la "Educación para todos".

La educación inclusiva está relacionada con la capacidad de potenciar y valorar la diversidad, entendiendo y protegiendo las particularidades de todos los sujetos, promoviendo el respeto a ser diferente, el aprender a vivir juntos y a garantizar la participación.

Según Blanco (2008), las características de la educación inclusiva en una institución educativa son:

-Inclusión

-Equidad

-Participación

-Diversidad

- Calidad

- Interculturalidad

- Carácter holístico

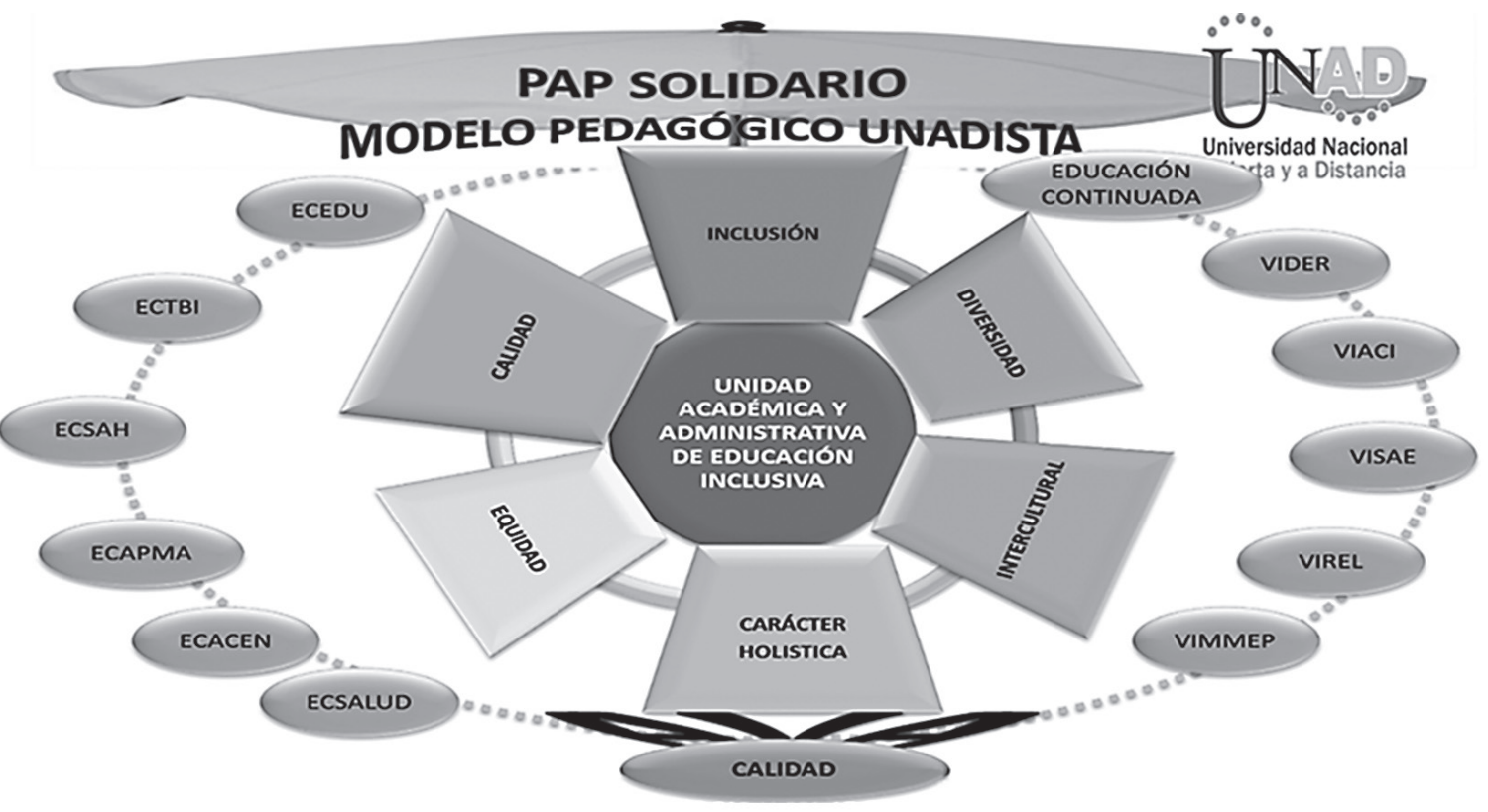

Gráfico 3. Características y modelo de educación inclusiva propuesto para la UNAD por Acevedo (2013). 


\section{Sujeto y construcción de conocimiento de poblaciones diversas}

El sujeto es el actor protagonista de las prácticas de reconocimiento y de conocimiento (Honnet, 2012), para la constitución subjetiva que se dan en los procesos educativos, por tanto no se trata tan sólo de brindar espacios para la participación. La educación inclusiva debe posibilitar a través de una pedagogía del reconocimiento, el despliegue subjetivo de los sujetos que viven esta experiencia que les permita reconstruirse y consolidar alternativas de futuro para sí mismo y para su comunidad.

Por tanto se refiere al reconocimiento de cada uno en relaciones intersubjetivas donde se viva la reciprocidad, en las cuales se supere la objetivación a partir de características con las cuales frecuentemente se nomina, para pasar a un verdadero conocimiento que permita visibilizar cómo se construye conocimiento en cada una de las poblaciones diversas tanto de la UNAD, como del país.

Es necesario indagar por los aprendizajes que se construyen en los encuentros desde la diferencia y a nivel de cada sujeto comprender cómo dinamizar procesos de autoaprendizaje y de metacognición para propiciar la permanencia de los estudiantes de las diversas poblaciones con el diseño de proyectos pedagógicos que permitan resolver las dificultades de acceso y generar diferentes maneras de circular en los ámbitos de la construcción del conocimiento.

Es importante que se resalte que la investigación en educación inclusiva es una apuesta por comprender cómo se puede dar el despliegue subjetivo en las múltiples potencialidades del sujeto en los procesos educativos, y cómo se pueden dinamizar prácticas de libertad a través de la configuración de espacios de expresión que permitan la visibilidad, con el fin de representar la existencia a través de la expresión artística en las diferentes formas ya sea en la música, la danza, la plástica, la escritura, el teatro, las artes visuales y en la recuperación de las formas tradicionales y ancestrales de expresión, en los diferentes entornos aprovechando los virtuales para propiciar la inclusión digital.

Es así como, el cuerpo se vuelve una categoría esencial y un escenario para la expresión del despliegue subjetivo, para propiciar la pedagogía del encuentro consigo mismo y con el otro para explorar las capacidades de aprendizaje, el universo cognitivo y las posibilidades existenciales de ser y de la experiencia de vivencia de la libertad.

Por tanto es importante investigar cómo se visibilizan y se diseñan propuestas pedagógicas de expresión artística, para propiciar el despliegue subjetivo, y así contribuir a la reconstrucción o reparación simbólica a través de experiencias y vivencias con el fin de configurar alternativas nuevas de ser y existir en el mundo. También permite la reconstrucción de culturas ancestrales de autocuidado y relación con la naturaleza en reconocimiento de cada sujeto en sus relaciones con el mundo y con los otros. 


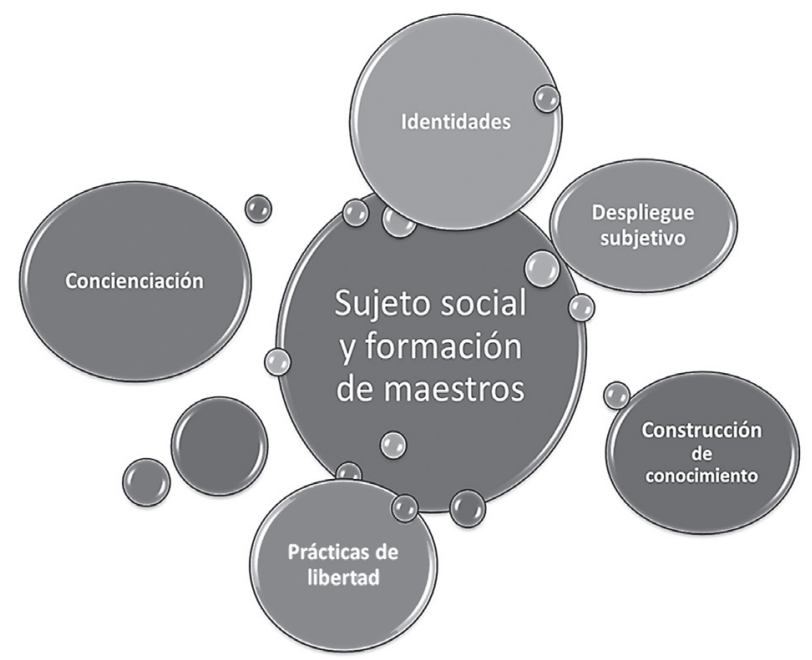

Gráfico 4. Categorías epistemológicas de la formación de maestros para la educación inclusiva (Acevedo,2014).

\section{Sujeto social y formación de maestros desde la educación inclusiva}

La apertura del sujeto hacia lo grupal se da en el marco de diferentes ámbitos, que le permiten configurar sus identidades, tales como la familia, la comunidad, su etnia, el grupo de trabajo, o por sus prácticas como es su cultura, su religión o las condiciones socioeconómicas, y en estos entornos es donde es posible que se produzcan nucleamientos que permitan la construcción del sujeto social, esto puede darse por necesidades, por un proyecto, una utopía, por tradición histórica o por un sueño de futuro.

Por tanto aquí se aborda la noción de identidad como forma de subjetividad en sentido de pertenencia colectiva, con signos compartidos, con memoria colectiva con lenguaje, prácticas cotidianas en espacios de acción y con trascendencia social (León y Zemelman, 1997).

Los desafíos de la formación de maestros, desde la educación inclusiva, implican necesariamente un abordaje epistemológico que posibilite prácticas de reconocimiento y prácticas de libertad (Freire, 1969). Superando por tanto el abordaje de que a los docentes tan solo se les debe sensibilizar, para pasar a otros niveles de profundidad en su formación para que el sujeto maestro se reconozca desde su propia historia como potenciador de la trasformación social, en los contextos y condiciones reales en las que se desenvuelva.

De esta manera, es necesario reconocerse como maestro en un proceso de concienciación que significa despertar la conciencia, con un cambio de mentalidad que implica la capacidad de analizar críticamente las causas y sus consecuencias para desarrollar una acción trasformadora con dignidad de ser sujeto en su momento histórico asumiendo prácticas de libertad. 


\section{La formación del maestro en la interculturalidad}

Así, formar maestros para la educación inclusiva de diversas poblaciones implica educar en y para la libertad, que supere la enseñanza para desarrollar la pedagogía del reconocimiento en la diversidad, la equidad y con una concepción del mundo de sus interacciones desde la interculturalidad en el empoderamiento.

La ECEDU busca formar a un maestro capaz de reflexionar sobre sus propias prácticas pedagógicas, e investigar sobre ellas para transformarlas en la interacción con los estudiantes y teniendo como referente la interculturalidad, tanto en el diseño de currículos, donde exista el principio de la accesibilidad y ésta se evidencie en la relación pedagógica, como acción coherente con el ejercicio y la defensa de derechos y la dignidad de todos los sujetos.

La relación pedagógica del reconocimiento requiere que se asuma como acción simbólica en la cual se materializan los propósitos pedagógicos, didácticos y formativos en los diferentes ambientes de aprendizaje.

Por tanto el maestro es aquel que reconoce y potencia el proceso de sujeto de una manera dinámica, comprendiendo las múltiples e infinitas formas de ser sujeto y las resistencias a los dispositivos de normalización, para favorecer las identidades movibles y transitorias que hoy se encuentran en los ambientes de aprendizaje (Infante, 2010).

\section{Inclusión digital y diseño de proyectos pedagógicos}

Para implementar la estrategia de inclusión digital, se requiere garantizar su coherencia con el discurso que sustenta el propósito, que es la inclusión social y la estrategia de la educación inclusiva. En primera medida, se requiere partir de un proceso de reconocimiento de los sujetos, y los contextos, en donde se desarrollará el diseño e implementación de un proyecto pedagógico, por lo que es fundamental realizar un proceso de identificación de necesidades que demuestre la pertinencia de la propuesta. Con base en lo anterior, la identificación de las necesidades de los sujetos tiene los siguientes elementos, lo que implica como mínimo los siguientes pasos:

a. Caracterización de sujetos y reconocimiento del contexto.

b. Recoger las voces y los relatos de los protagonistas.

c. Encontrar las necesidades más sentidas por los diferentes actores de la comunidad escolar.

d. Configurar el campo de discusión con un estado del arte.

e. Identificar las condiciones y posibilidades tecnológicas de la población y la cultura.

f. Encontrar alternativas de solución dentro del mismo contexto con nuevas interpretaciones. 


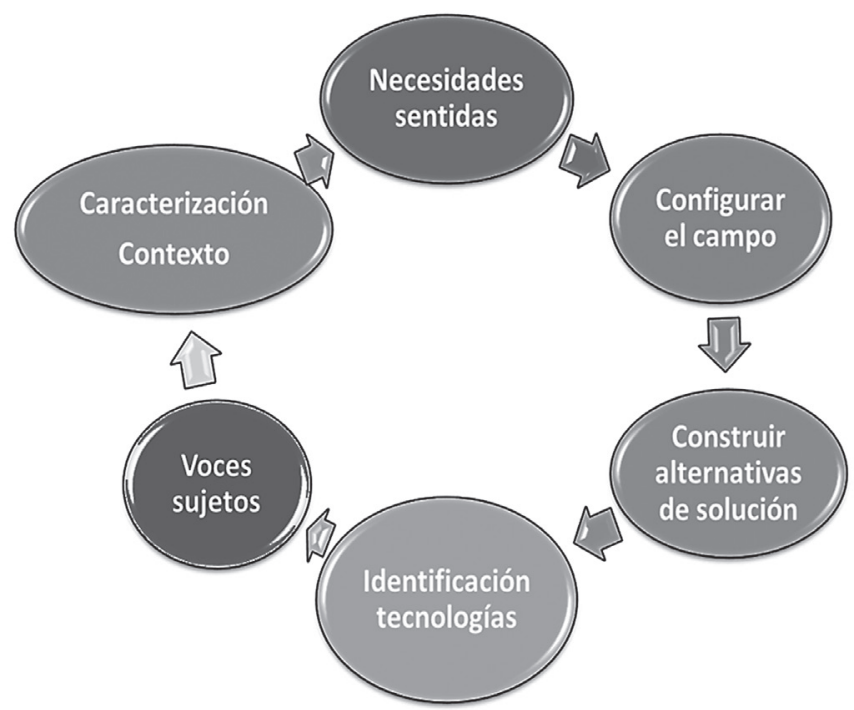

Gráfico 5. Pasos en la identificación de las necesidades para proyecto pedagógico con inclusión digital (Acevedo, 2014).

\section{El papel de las tecnologías de la comunicación en el diseño de innovaciones pedagógicas con el uso del diseño universal}

La sociedad ha venido imponiendo múltiples demandas comunicativas a las instituciones de educación, a través de los estudiantes, quienes son sujetos con identidades múltiples, que surgen en contextos complejos y diversos. Por lo anterior se hace inevitable la apropiación de las tecnologías de la información y la comunicación, para responder a las necesidades comunicativas y de aprendizaje de todos los sujetos de las diversas poblaciones.

En este sentido, Echeita y Ainscow (2010) plantean que en el diseño pedagógico es necesario realizar un proceso de programación didáctica, para valorar la diversidad de los estudiantes y tener presente, por ello, las necesidades educativas en coherencia con los principios de inclusión educativa.

El diseño de innovaciones pedagógicas, desde la perspectiva de la educación inclusiva y de la inclusión digital, se puede materializar en el Diseño Universal para el Aprendizaje -DUA-, que integra el marco educativo y tecnológico, de naturaleza práctica, capaz de maximizar las oportunidades de aprendizaje de todos los estudiantes.

En el DUA se hace indispensable la incorporación de las tecnologías y de las propuestas pedagógicas en el currículo, con elementos alternativos que garanticen que sea accesible a todos los estudiantes con necesidades de aprendizaje múltiples y diversas. 
Se retoman aquí algunas consideraciones sobre la experiencia del Centro para la Tecnología Especial y Aplicada -CAST (2008), el cual en los últimos 25 años se ha centrado en desarrollar ayudas con el uso de las tecnologías para adaptar a las personas con discapacidad al currículo; éste proceso llevó a determinar que el currículo general y estandarizado puede limitar aún más a esas personas con discapacidad en su aprendizaje.

En los años 90 el foco de atención cambió hacia el currículo general y sus limitaciones, llegando a la conclusión de que la discapacidad está en el currículo y no en las personas, es decir, son los currículos los que no están diseñados para adaptarse a las diferencias individuales.

En consecuencia el CAST «empezó a investigar, desarrollar y articular los principios y las prácticas del Diseño Universal para el Aprendizaje» (CAST, 2008). Éste concepto tiene su fuente inicial en el DUA, el cual consiste en el diseño y construcción de espacios accesibles para todas las personas (diseño para todos), lo cual implica que a la hora de diseñar es necesario tener todas las precauciones posibles para garantizar el acceso a todo público y así evitar posteriormente adaptaciones de los espacios.

\section{Un proyecto pedagógico implica asumir el diseño curricular con el uso de las tecnologías de la comunicación}

El DUA, basado en los planteamientos del diseño para todos, es una postura que se enfrenta directamente con las barreras que el currículo inflexible le impone al aprendizaje, al estar diseñado sin tener en cuenta las necesidades de todos los sujetos y su diversidad.

Es primordial definir el currículo desde el marco del DUA, el cual se compone de cuatro aspectos fundamentales que deben estar articulados y entrelazados:

1. Metas: puntos de referencia o expectativas visualizadas en las competencias o habilidades a alcanzar.

2. Métodos: métodos instructivos específicos desarrollados por el profesor.

3. Materiales: medios y materiales usados para enseñar y aprender.

4. Evaluación: razones y métodos que se usan para valorar el progreso de los estudiantes.

El currículo como concepto del DUA puede estar sistemáticamente diseñado desde el principio, con dos fines, por un lado que se reduzcan las adaptaciones curriculares a posteriori y por otro que se implemente un medio de aprendizaje mejor para todos los estudiantes. 
En éste sentido el diseño de un proyecto pedagógico luego de identificar las necesidades de los sujetos y reconocer el contexto debe tener un claro modelo pedagógico y definir claramente los propósitos de formación y su currículo debe asumir el desafío de atender los requerimientos de las poblaciones diversas, por tanto la enseñanza y los procesos de aprendizaje se deben diseñar en perspectiva de multinivel partiendo inicialmente de la necesidades de los sujetos y apoyarse en los principios de un diseño universal que garantice la educación para todos.

Como vemos el DUA, se ha beneficiado de las tecnologías para individualizar el currículo en la práctica, de manera más fácil y efectiva, debido a la flexibilidad que brindan las tecnologías, lo cual tiene efecto en el proceso pedagógico a través de la adaptación del currículo los cuales pueden diseñar e implementar ambientes personalizados a los medios digitales para responder a la necesidades y referencias de los estudiantes y de las diversas poblaciones que lo requieran.

Para el CAST el DUA obedece a tres principios operativos:

- Minimizar las barreras de aprendizaje, a través de la multiplicidad de formas de representación de los contenidos, abriendo un amplio espectro de opciones para el acceso real al aprendizaje.

- Proporcionar múltiples medios de expresión al estudiante, para que pueda demostrar lo aprendido de acuerdo a su propio estilo y preferencia.

- Multiplicidad de medios para su motivación, ayudando a encontrar el significado de los propósitos de formación, así como propiciar acciones de motivación para que el estudiante encuentre un incentivo personal hacia el aprendizaje.

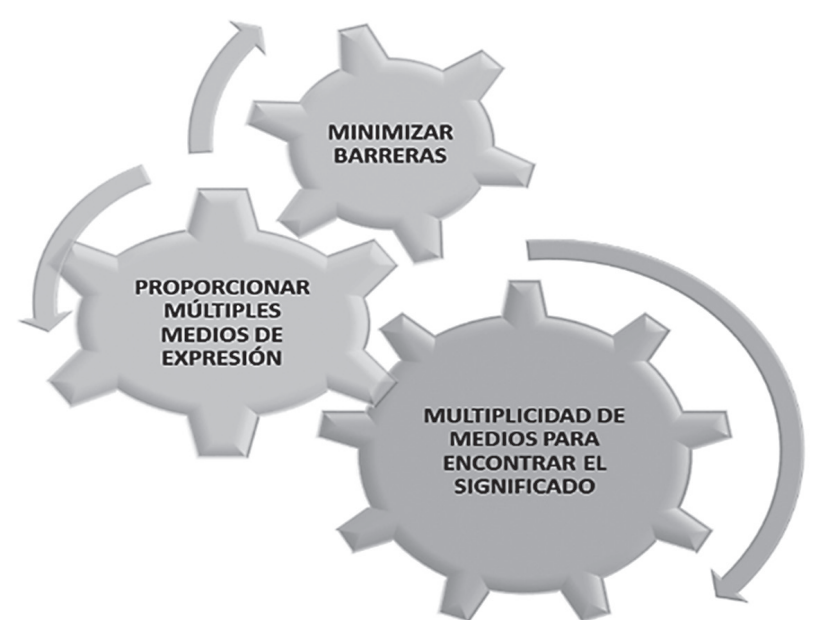

Gráfico 6. Principios operativos del Diseño Universal para el Aprendizaje (Acevedo,2014). 
Burgstahler (2007) citado por Bou Gerena (2011), organizó los principios del DUA en ocho categorías como indicadores que se han adaptado en este escrito con un enfoque general:

- Climas de la comunicación: adoptar y proponer prácticas que reflejen los valores más altos respecto a la diversidad y la inclusión.

- Interacción: fomentar la interacción regular eficaz entre estudiante y maestro, y asegurarse de que los métodos de comunicación sean accesibles a todos los estudiantes.

- Entornos físicos, virtuales y productos: asegurar que las instalaciones, actividades, materiales y equipos sean físicamente accesibles y utilizables por todos los estudiantes y que se aborden todas las características potenciales de los estudiantes considerando los aspectos para su seguridad. - Modelos de enseñanza: uso de modelos de enseñanza claros y bien fundamentados que sean accesibles para todos los estudiantes.

- Recursos de información y tecnología: garantizar que los materiales del curso, las anotaciones y otros recursos de información sean atractivas, flexibles y accesibles para todos los estudiantes en los diferentes entornos. - Retroalimentación: proporcionar regularmente tiempo para la retroalimentación.

- Evaluación: evaluar los progresos del estudiante regularmente utilizando varias técnicas de accesibilidad y herramientas, revisar y ajustar las recomendaciones para los estudiantes.

- Acomodo: planear los procesos por medio de los cuales se da la apropiación de las dinámicas pedagógicas de acuerdo con el reconocimiento de los estudiantes y aproximarse a sus necesidades para que sean satisfechas por el diseño de la innovación en el proceso mismo de implementación.

\section{Recomendaciones sobre los elementos tecnológicos en el diseño de entornos virtuales en páginas web para la educación inclusiva}

Para Colombia se ha creado la norma técnica número 5854, sobre accesibilidad a páginas Web. Esta norma tiene por objeto establecer los requisitos de accesibilidad que se deben implementar en las páginas web en los niveles de conformidad A, AA y AAA.

Los requisitos de la norma se alinean por principios:

- Principio 1: Perceptible

- Principio 2: Operable

- Principio 3: Comprensible

- Principio 4: Robusto 


\section{Principio 1: Perceptible}

La información y los componentes de la interfaz de usuario deben presentarse a los usuarios, de modo que ellos puedan percibirlos.

\section{-Alternativas textuales}

Se deben proporcionar alternativas textuales para todo contenido no textual, de modo que se pueda convertir a otros formatos que las personas necesiten, tales como textos ampliados, braille, voz, símbolos o en un lenguaje más simple.

\section{-Adaptable}

Se debe crear contenidos que puedan presentarse de diferentes formas (por ejemplo, con una disposición más simple), sin perder información o estructura.

\section{-Distinguible}

Se debe facilitar a los usuarios ver y oír el contenido, incluyendo la separación entre el primer plano y el fondo.

\section{Principio 2: Operable}

Los componentes de la interfaz de usuario y la navegación deben ser operables.

-Accesible por teclado

Se debe proporcionar acceso a toda la funcionalidad mediante el teclado.

\section{-Tiempo suficiente}

Se debe proporcionar a los usuarios el tiempo suficiente para leer y usar el contenido.

\section{-Navegable}

Se debe proporcionar medios para ayudar a los usuarios a navegar, encontrar contenido y determinar dónde se encuentran.

\section{Principio 3: Comprensible}

La información y el manejo de la interfaz de usuario deben ser comprensibles.

$$
\text { -Legible }
$$

Se debe hacer que los contenidos textuales resulten legibles y comprensibles.

\section{-Predecible}

Se debe hacer que las páginas web aparezcan y operen de manera predecible.

$$
\text { -Entrada de datos asistida }
$$

Se debe ayudar a los usuarios a evitar y corregir errores. 


\section{Principio 4: Robusto}

El contenido debe ser suficientemente robusto como para ser interpretado de forma fiable por una amplia variedad de aplicaciones de usuario, incluyendo las ayudas técnicas.

\section{-Compatible}

Se debe maximizar la compatibilidad con las aplicaciones de usuario actuales y futuras, incluyendo las ayudas técnicas.

\section{-Conformidad}

En esta sección se presentan los requisitos de conformidad con las WCAG 2.0. (Guías de Accesibilidad a contenidos Web). También se proporciona información acerca de cómo realizar declaraciones de conformidad, las cuales son opcionales.

\section{Requisitos de conformidad}

Para que una página web sea certificada de acuerdo con la presente norma, deben satisfacerse todos los requisitos siguientes:

\section{-Nivel de conformidad}

Para lograr conformidad con el Nivel A (el mínimo), la página web debe satisfacer todos los Criterios de Conformidad del Nivel A. De la misma manera se logra la conformidad en los niveles AA o AAA.

\section{-Páginas completas}

La conformidad (y los niveles de conformidad) se deben aplicar a las páginas web completas, y no pueden ser alcanzadas, si se excluye una parte de la misma.

\section{-Procesos completos}

Cuando una página web forma parte de una serie de páginas web que presentan un proceso (es decir, una secuencia de pasos que es necesario completar para realizar una actividad), todas las páginas en ese proceso, deben ser conformes con el nivel especificado o uno superior. (No es posible lograr conformidad con un nivel en particular si una de las páginas del proceso no cumple con ese nivel o uno superior).

\section{Declaraciones de conformidad}

La conformidad con la norma se aplica sólo a las páginas web. Sin embargo, la certificación de conformidad puede cubrir una sola página, una serie de páginas o múltiples páginas web relacionadas. 
Inclusión digital y educación inclusiva. Aportes para el diseño de proyectos pedagógicos con el uso de tecnologías de la comunicación. Artículo de reflexión.

\section{Referencias bibliográficas}

_1994. Declaración de Salamanca. Disponible en: http://www.unesco.org/education/ pdf/SALAMA_S.PDF Consultado el 01/05/2012.

Acevedo, S. 2014. Conferencia Inclusión en pedagogía y tecnologías de la comunicación. En Universidad de la Guajira. Conferencista en el Primer Coloquio sobre pedagogía y apropiación significativa de tecnologías de la información y la comunicación. Riohacha.

Acevedo, S. 2013. Configuración de la línea de investigación en Visibilidad, Gestión del Conocimiento y Educación Inclusiva. Bogotá: UNAD- CETICS.

Blanco, R. 2008. La educación inclusiva: el camino hacia el futuro. Conferencia Internacional de educación agosto del 2008. Ginebra.

Bou Gerena, C. 2011. Diseño universal para el aprendizaje (DUA) como Herramienta para Inclusión. Consultado en: http://www.suagm.edu/umet/biblioteca/UMTESIS/ Tesis_Educacion/Educacion_especial_2011/CBou\%2012-12-11\%20\%28SEGURO\%29.pdf

Carrillo, L. 2014. Conferencia Educación inclusiva. En: Seminario Internacional de Educación Inclusiva y Tecnologías de la Comunicación. UNAD. Disponible en: http:// www.youtube.com/watch?v=-ccMc2-cwdc 20 de febrero de 2014.

Delors, J. 1996. L'éducation un trésor est cachédedans, Rapport à l'UNESCO de la Commission internationale sur l'éducation pour le vingt et unième siècle, Pp. 83-96. UNESCO.

Echeita, G \& Ainscow, M. 2010. Conferencia Un marco de referencia y pautas de acción para el desarrollo de sistemas de educación incluyentes. En el II Congreso Iberoamericano de Síndrome de Down, organizado por Down España, celebrado en Granada en mayo de 2010.

Fernández, C et al. 2012. Inclusión un acontecimiento para todos. Comisiones de educación y empoderamiento y de juventud e inclusión de la red argentino-americana para el liderazgo (REAL). IVLP Alumni Network of the Americas -IANA Diciembre 2012. http://www.ianamericas.org/wp-content/uploads/2012/12/exclusion.jpg

Freire, P. 1971. Educación como práctica de la libertad. México: Siglo XXI Editores.

Freire, P. 1969. Pedagogía del oprimido. México: Siglo XXI Editores.

Honnet, A. 2012. Reificación. Un estudio en la teoría del reconocimiento. Buenos Aires: Katz editores.

Infante, M. 2010. Desafios a la formación docente: inclusión educativa REVISIONES. En Estudios Pedagógicos XXXVI, № 1: 287-297, 2010 Santiago de Chile. 
León, E \& Zemelman, H. 1997. Subjetividad: umbrales de pensamiento social. Barcelona: Anthropos.

López, N. 2012. Equidad educativa y diversidad cultural en América Latina - la ed. Ciudad Autónoma de Buenos Aires: Instituto Internacional de Planeamiento de la Educación IIPE-Unesco.

Ministerio de Educación Nacional (MEN). 2013. Lineamientos de Política de Educación Superior Inclusiva. Bogotá: MEN.

National Center on Universal Desing for Learning. 2008. UDL Guidelines version 1.0. Aapectos definidos por el CAST 2008 Centro para la Tecnología Especial y Aplicada 2008. Consultado en mayo de 2014. En: http://web.uam.es/personal_pdi/stmaria/sarrio/ DOCENCIA/ASIGNATURA\%20BASES/LECTURAS\%20ACCESIBLES\%20Y\%20GUIONES\%20 DE\%20TRABAJO/Diseno\%2oUniversal\%20de\%20Aprendizaje.pdf

Opertti, R. 2008. Inclusión educativa: el camino del futuro. Un desafio para compartir. En: 48a reunión de la Conferencia Internacional de Educación (CIE), UNESCO, Ginebra.

Parrilla, A. 2002. Acerca del origen y sentido de la educación inclusiva. En Revista de Educación, 327. (pp 11-29).

Sanders, T. 1968. The Paulo Freire Method. New York: American Universities Field Staff.

UNESCO. 2005. Convención sobre la protección y promoción de la diversidad de las expresiones culturales, París. Disponible en: http://unes-doc.unesco.org/ images/0014/001429/142919s.pdf consultado en junio de 2010.

Urresti, M. 2000. Adolescencia y juventud: dos categorías construidas socialmente. En: E. Tenti Fanfani (comp.), Una escuela para los adolescentes. Reflexiones y valoraciones. Buenos Aires: UNICEF-Losada. 